\title{
FRAGMENT OF NONSTANDARD ANALYSIS WITH A FINITARY CONSISTENCY PROOF
}

\author{
MICHAL RÖSSLER AND EMIL JEŘÁBEK
}

\begin{abstract}
We introduce a nonstandard arithmetic $\mathrm{NQA}^{-}$based on the theory developed by R. Chuaqui and P. Suppes in [2] (we will denote it by $\mathrm{NQA}^{+}$), with a weakened external open minimization schema. A finitary consistency proof for $\mathrm{NQA}^{-}$formalizable in PRA is presented. We also show interesting facts about the strength of the theories NQA and $\mathrm{NQA}^{+} ; \mathrm{NQA}^{-}$is mutually interpretable with $\mathrm{I}_{0}+\mathrm{EXP}$, and on the other hand, $\mathrm{NQA}^{+}$ interprets the theories $I \Sigma_{1}$ and $W K L_{0}$.
\end{abstract}

$\S 1$. Introduction. In the early 1960's, Abraham Robinson [9] showed that there is a rigorous foundation for the use of infinitesimals in mathematical analysis. This development of differential and integral calculus was called nonstandard analysis. Robinson's original approach is based on construction of nonstandard extensions by ultraproducts.

His successors introduced different frameworks for development of the nonstandard analysis, see for instance Nelson's internal set theory [7], Ballard and Hrbáček [1], Vopěnka's alternative set theory [15], and Chuaqui and Suppes $[12,2]$. Chuaqui and Suppes $[12,2]$ introduced a weak nonstandard theory (we denote it by $\mathrm{NQA}^{+}$) suitable for developing a fundamental part of analysis. They also presented that the proposed system of axioms has finitary consistency proof.

There are many interpretations of the finitism of Hilbert. We show that $\mathrm{WKL}_{0}$ and $I \Sigma_{1}$ are interpretable in the system of Chuaqui and Suppes, hence it is not finitistic in sense of Tait [13].

Unfortunately, the consistency proof of Chuaqui and Suppes contains some opacities and a logical gap. The goal of the proof is to interpret terms of the system as expressions of the form

$$
\frac{a_{0} x^{r_{0}}+a_{1} x^{r_{1}}+\cdots+a_{n} x^{r_{n}}}{b_{0} x^{s_{0}}+b_{1} x^{s_{1}}+\cdots+b_{n} x^{s_{m}}}
$$

where $a_{i}, b_{j}, r_{i}, s_{j}$ are positive rational numbers and $x$ interprets a nonstandard integer $\nu_{0}$. However, this interpretation is not well adapted to the exponential terms (e.g. $2^{\nu_{0}}$ ), if the equality axioms for these terms are required to be satisfied.

The authors avoid the problems by omitting the equality axioms for recursively defined functions. On page 131 the authors state:

We know from Herbrand's theorem that the theory is inconsistent if and only if there is a conjunction of closed substitution instances of the axioms which is inconsistent. We also have to include instances of the equality axioms for the field operations. The corresponding equality 
theorems for the operations defined by recursion are proved by induction, so that we do not need to include their instances.

Although the induction is not an axiom there, it is a theorem provable from the minimum axiom and equality axioms (not only for field operation but for all equality axioms). Therefore, if instances of equality axiom for operations defined by recursion are not included, the induction cannot be proved.

The aim of this paper is to introduce a new theory $\mathrm{NQA}^{-}$based on the system presented in [2] and prove the finitary consistency of our theory. Actually, our theory has consistency proof formalizable in PRA. Therefore it is more suitable to satisfy a part of Hilbert's program of proof theory in strict meaning of Tait.

This paper is organized into four sections. In the second section, we present the language and axioms of the theories $\mathrm{NQA}^{+}$and $\mathrm{NQA}^{-}$. Both these theories are open and nonstandard extensions of the theory of ordered fields with a built-in arithmetic of natural numbers. These theories are sufficiently strong to develop a basic infinitesimal calculus and sufficiently weak to prove their consistency formally in some arithmetic.

In the third section it is shown that $\mathrm{NQA}^{-}$has a finitary proof of consistency. The proof can be formalized in the primitive recursive arithmetic PRA or in $I \Sigma_{1}$. The Hilbert-Ackermann Theorem is used in the proof. An algorithm for the construction of a finite model from a finite closed axiom instances can be obtained from the proof.

In the fourth section we study relations between NQA ${ }^{-}$and NQA ${ }^{+}$. Both theories are compared from the arithmetical point of view. We show that $\mathrm{NQA}^{-}$and $\mathrm{I} \Delta_{0}+$ EXP mutually interpret each other and the theories $W K L_{0}$ and $I \Sigma_{1}$ are both interpretable in $\mathrm{NQA}^{+}$.

\section{§2. The theories $\mathrm{NQA}^{-}$and $\mathrm{NQA}^{+}$.}

2.1. The language of the theories $\mathrm{NQA}^{-}$and $\mathrm{NQA}^{+}$. We will work in a first-order language with equality where the variables range over numbers.

Further we suppose that the language contains the following basic symbols:

1. symbols for the constants 0,1 ;

2. a symbol $\nu_{0}$ for the constant of a nonstandard natural number;

3. a binary predicate symbol $x<y$ for the linear ordering;

4. a unary predicate symbol $\mathbb{N}(x)$ - "to be a natural number";

5. a unary predicate symbol $\operatorname{Inf}(x)$ - "to be infinitesimal";

6. binary function symbols $x+y, x-y, x \cdot y$ and $x / y$ for basic field operations;

7. a unary function symbol $\delta(x)$ for the sign function;

8. a binary function symbol $x^{y}$ for the power (exponential) function*;

9. unary function symbol $x$ ! for the factorial ${ }^{\dagger}$;

10. unary function $\operatorname{symbol} \operatorname{li}(x)$ for the ceiling function ${ }^{\ddagger}$.

\footnotetext{
*As we will see later, the theory contains an axiom only for powers with natural exponent.

$\dagger$ Also the factorial axiom is included only for the factorial of a natural number.

${ }^{\ddagger}$ In fact, it is not exactly the ceiling function, but only a function giving a natural number greater or equal than its argument. The ceiling function could be defined using a minimum operator as $\lceil y\rceil \stackrel{\text { def }}{=} \min _{x \geq y}(y)$. The function li $(y)$ guarantee that the formula $x \geq y$ is satisfiable.
} 
Definition 2.1. A formula $\varphi$ is called internal if there is no occurrence of the predicate Inf in it. A formula containing the predicate Inf is called an external formula.

For simplification, we will use the notation $\vec{y}=\left(y_{1}, y_{2}, \ldots, y_{m}\right)$. In our language we also have recursive operators on the existing terms and formulas:

11. the operator ${ }^{\S} \min _{\varphi}$ - denoted also by $\mu x[\varphi(x, \vec{y})](\vec{y})$-defined only for an open formula $\varphi(x, \vec{y})$ in which no other min occurs,

12. operators $\max _{\tau}$ and $\sum \tau$ defined for any term $\tau(x, \vec{y})$ without any min occurring in the term $\tau(x, \vec{y})$.

We denote thWe denote the language without any min-term by symbol $\mathrm{L}^{\varnothing}$. The full language (with $\min _{\varphi}$ function symbols) we denote by $\mathrm{L}$.

By the operator $\max _{\tau}$ we mean the minimal index less or equal to the argument where the term $\tau$ reaches the maximum. For better understanding, we show a few examples.

EXAmple. $\quad$ 1. For $\tau=\frac{n^{2}-1}{n !}$ we have $\max _{\tau}(n)=\left\{\begin{array}{ll}0 & \text { for } n<2 \\ 2 & \text { for } n \geq 2\end{array}\right.$;

2. For $\tau=n$ we have $\max _{\tau}(n)=n$;

3. For $\tau=n$ ! we have $\max _{\tau}(n)=\left\{\begin{array}{ll}0 & \text { for } n<2 \\ n & \text { for } n \geq 2\end{array}\right.$.

Definition 2.2. We say that a term $\sigma$ occurs recursively in a term $\tau$ (or in a formula $\varphi$ ) if

1. $\sigma$ is a subterm of $\tau$ or $\varphi$, or

2. there is a subterm of form $\sum \varrho, \max _{\varrho}$, or $\min _{\psi}$, such that $\sigma$ occurs recursively in a term $\varrho$ (or in a formula $\psi)$.

e language without any min-term by symbol $L^{\varnothing}$. The full language (with $\min _{\varphi}$ function symbols) we denote by $\mathrm{L}$.

The operator $\max _{\tau}$ has a meaning of the minimal index less or equal to the argument where the term $\tau$ reaches the maximum. For better understanding, we show a few examples.

EXAmple. $\quad$ 1. For $\tau=\frac{n^{2}-1}{n !}$ we have $\max _{\tau}(n)=\left\{\begin{array}{ll}0 & \text { for } n<2 \\ 2 & \text { for } n \geq 2\end{array}\right.$;

2. For $\tau=n$ we have $\max _{\tau}(n)=n$;

3. For $\tau=n$ ! we have $\max _{\tau}(n)=\left\{\begin{array}{ll}0 & \text { for } n<2 \\ n & \text { for } n \geq 2\end{array}\right.$.

Definition 2.3. We say that a term $\sigma$ occurs recursively in a term $\tau$ (or in a formula $\varphi$ ) if

1. $\sigma$ is a subterm of $\tau$ or $\varphi$, or

2 . there is a subterm of form $\sum \varrho, \max _{\varrho}$, or $\min _{\psi}$, such that $\sigma$ occurs recursively in a term $\varrho$ (or in a formula $\psi)$.

$\S$ Function symbols $\min _{\varphi}$ are Skolem functions of the open induction. Note that $\min _{\varphi}$ can simulate the existential quantifier and to prevent this in another minimum we require that no other $\min _{\psi}$ is occurring in the formula $\varphi$. 
2.2. The axioms of the theories $N Q A^{-}$and $N Q A^{+}$. The theories $N Q A^{-}$ and $\mathrm{NQA}^{+}$are open first-order theories with equality. These theories contain the following axioms.

1. The ordered field axioms OF-in the form of open axioms using the functions $x-y$ and $x / y$.

2. Robinson's arithmetic $Q$ relativized to the predicate $\mathbb{N}$ (especially $\mathbb{N}(\bar{n})$ for any numeral $\bar{n}$, we understand by the symbol $\bar{n}$ the term $\underbrace{1+1+\ldots+1}_{n \text {-times }})$.

3. The corresponding axioms for the non-basic arithmetical functions (sign, power, factorial and li function)

Axiom 1 (FC1). $(x \geq 0 \rightarrow \delta(x)=1) \&(x<0 \rightarrow \delta(x)=-1)$,

Axiom 2 (FC2). $\left(x^{0}=1\right) \&\left(\mathbb{N}(n) \rightarrow x^{n+1}=x^{n} \cdot x\right)$,

Axiom 3 (FC2). $(0 !=1) \&(\mathbb{N}(n) \rightarrow(n+1) !=n ! \cdot(n+1))$,

Axiom 4 (FC3). $\mathbb{N}(\operatorname{li}(x)) \&(\operatorname{li}(x) \geq x)$.

Note that with a help of the sign function, we can define the absolute value function as

$$
|x| \stackrel{\text { def }}{=} x \cdot \delta(x)
$$

4. The system of infinitesimal axioms for the predicate Inf

Axiom 5 (IF1). $\nu_{0} \neq 0 \& \mathbb{N}\left(\nu_{0}\right) \& \operatorname{Inf}\left(1 / \nu_{0}\right)$,

Axiom $6($ IF2). $(\operatorname{Inf}(x) \&|y| \leq|x|) \rightarrow \operatorname{Inf}(y)$,

Axiom 7 (IF3). $(\operatorname{Inf}(x) \& \neg \operatorname{Inf}(1 / y)) \rightarrow \operatorname{Inf}(x \cdot y)$,

Axiom 8 (IF4). $(x \neq 0 \& \operatorname{Inf}(x)) \rightarrow \neg \operatorname{Inf}(1 / x)$,

Axiom 9 (IF5). $(\neg \operatorname{Inf}(1 / x) \& \neg \operatorname{Inf}(1 / y)) \rightarrow \neg \operatorname{Inf}(1 /(x+y))$.

Originally, there were two more axioms in [2], but they are provable from the others. From the Inf predicate and axioms of infinitesimal calculus, described above, we can define correctly the predicate "to be a standard natural number" as

$$
\mathbb{F} \mathbb{N}(x) \stackrel{\text { def }}{=}(\mathbb{N}(x) \& \neg \operatorname{Inf}(1 /(x+1))) .
$$

5. Recursion axioms for the operators $\max _{\tau}$ and $\sum \tau$

Axiom $10(\mathrm{MAX})$. Let $\tau(x, \vec{y})$ be an $(m+1)$-ary term in the language $\mathrm{L}^{\varnothing}$. Then the term $\max _{\tau}(n, \vec{y})$ satisfies

$$
\begin{gathered}
\max _{\tau}(0, \vec{y})=0, \\
\left(\mathbb{N}(n) \& \tau(n+1, \vec{y}) \leq \tau\left(\max _{\tau}(n, \vec{y}), \vec{y}\right)\right) \rightarrow \max _{\tau}(n+1, \vec{y})=\max _{\tau}(n, \vec{y}), \\
\left(\mathbb{N}(n) \& \tau(n+1, \vec{y})>\tau\left(\max _{\tau}(n, \vec{y}), \vec{y}\right)\right) \rightarrow \max _{\tau}(n+1, \vec{y})=n+1
\end{gathered}
$$

Axiom 11 (SUM). Let $\tau(x, \vec{y})$ be an $(m+1)$-ary term in the language $\mathrm{L}^{\varnothing}$. Then the term $\sum_{i=0}^{n} \tau(i, \vec{y})$ satisfies

$$
\sum_{i=0}^{0} \tau(i, \vec{y})=\tau(0, \vec{y}) \&\left(\mathbb{N}(n) \rightarrow \sum_{i=0}^{n+1} \tau(i, \vec{y})=\sum_{i=0}^{n} \tau(i, \vec{y})+\tau(n+1, \vec{y})\right)
$$


6. The open internal minimum axiom O-MIN for the operator $\min _{\varphi}$

Axiom $12(\mathrm{O}-\mathrm{MIN})$. Let $\varphi(x, \vec{y})$ be an open internal formula in the language $\mathrm{L}^{\varnothing}$. Then the term $\min _{\varphi}(\vec{y})$ satisfies

$$
(\mathbb{N}(x) \& \varphi(x, \vec{y})) \rightarrow\left(\mathbb{N}\left(\min _{\varphi}(\vec{y})\right) \& \min _{\varphi}(\vec{y}) \leq x \& \varphi\left(\min _{\varphi}(\vec{y}), \vec{y}\right)\right) .
$$

7. The open weak external minimum axiom $\mathrm{O}-\mathrm{MIN}^{\mathrm{n}}$ for the operator $\min _{\varphi}$

Axiom $13\left(\mathrm{O}-\mathrm{MIN}^{\mathrm{n}}\right)$. Let $\varphi(x, \vec{y})$ be an open external formula in the language $\mathrm{L}^{\varnothing}$ and $\bar{n}$ be a numeral. Then for the term $\min _{\varphi}(\vec{y})$, we have

$$
\varphi(\bar{n}, \vec{y}) \rightarrow\left(\mathbb{N}\left(\min _{\varphi}(\vec{y})\right) \& \min _{\varphi}(\vec{y}) \leq \bar{n} \& \varphi\left(\min _{\varphi}(\vec{y}), \vec{y}\right)\right)
$$

8. The open external minimum axiom O-MIN ${ }^{\text {st }}$ for the operator $\min _{\varphi}$

AxIOM $14\left(\mathrm{O}-\mathrm{MIN}^{\text {st }}\right)$. Let $\varphi(x, \vec{y})$ be an open external formula in the language $L^{\varnothing}$. Then for the term $\min _{\varphi}(\vec{y})$, we have

$$
(\mathbb{F} \mathbb{N}(x) \& \varphi(x, \vec{y})) \rightarrow\left(\mathbb{N}\left(\min _{\varphi}(\vec{y})\right) \& \min _{\varphi}(\vec{y}) \leq x \& \varphi\left(\min _{\varphi}(\vec{y}), \vec{y}\right)\right) .
$$

By $\mathrm{NQA}^{\varnothing}$ we denote the theory in the language $\mathrm{L}^{\varnothing}$ and with the axioms above except any minimum schema.

Then we can introduce the theories $\mathrm{NQA}^{+}$and $\mathrm{NQA}^{-}$in the language $\mathrm{L}$ as

- $\mathrm{NQA}^{+}=\mathrm{NQA}^{\varnothing}+\mathrm{O}-\mathrm{MIN}+\mathrm{O}-\mathrm{MIN}^{\mathrm{st}}$,

- $N Q A^{-}=N Q A^{\varnothing}+O-M I N+O-M^{n} N^{n}$.

2.3. Equivalent axioms for $\mathrm{NQA}^{-}$. The axioms introduced above are not the only one characterization of the theories $\mathrm{NQA}^{+}$and $\mathrm{NQA}^{-}$. In this section we introduce simpler, but equivalent versions of the $\mathrm{NQA}^{-}$theory.

Definition 2.4. We introduce bounded quantifiers as follows

1. $(\exists x \leq \tau(y)) \varphi(x, y) \stackrel{\text { def }}{\equiv}(\exists x)(x \leq \tau(y) \& \mathbb{N}(x) \& \varphi(x, y))$

2. $(\forall x \leq \tau(y)) \varphi(x, y) \stackrel{\text { def }}{\equiv}(\forall x)((x \leq \tau(y) \& \mathbb{N}(x)) \rightarrow \varphi(x, y))$.

A formula $\varphi$ is said to be a $\Delta_{0}$-formula if all quantifiers in $\varphi$ are bounded.

Our main tool is the following lemma, which makes it possible to construct a characteristic function $\chi_{\varphi}$ for every internal $\Delta_{0}$-formula $\varphi$ without using minfunctions.

LEMmA 2.5. Let $\varphi(\vec{x})$ be an internal $\Delta_{0}$-formula. Then there is a term $t_{\varphi}(\vec{x})$ in the language of $\mathrm{NQA}^{\varnothing}$ such that $\mathrm{NQA}^{-}$proves

$$
\left(\varphi(\vec{x}) \leftrightarrow t_{\varphi}(\vec{x})=1\right) \&\left(\neg \varphi(\vec{x}) \leftrightarrow t_{\varphi}(\vec{x})=0\right)
$$

Proof. We proceed by induction on the complexity of the formula $\varphi$. First, for atomic formulae, we introduce a new function $\theta(x)$ given by

$$
\theta(x) \stackrel{\text { def }}{=} \frac{1}{2}(\delta(x)+\delta(-x))= \begin{cases}1 & x=0 \\ 0 & x \neq 0\end{cases}
$$

Then it is easy to see that

$$
\begin{aligned}
& \tau(x)=\sigma(x) \leftrightarrow(\tau(x)-\sigma(x))=0 \leftrightarrow \theta(\tau(x)-\sigma(x))=1, \text { and } \\
& \tau(x) \neq \sigma(x) \leftrightarrow(\tau(x)-\sigma(x)) \neq 0 \leftrightarrow \theta(\tau(x)-\sigma(x))=0 .
\end{aligned}
$$


Therefore, we define

$$
\chi_{[\sigma(\vec{x})=\tau(\vec{x})]}(\vec{x}) \stackrel{\text { def }}{=} \theta(\tau(\vec{x})-\sigma(\vec{x})) .
$$

On the other hand, we easily get that

$$
\begin{aligned}
\tau(x)<\sigma(x) & \leftrightarrow(\tau(x)-\sigma(x))<0 \leftrightarrow \delta(\tau(x)-\sigma(x))=-1 \leftrightarrow \\
& \leftrightarrow 1-\frac{1}{2}(1+\delta(\tau(x)-\sigma(x)))=1, \text { and } \\
\tau(x) \geq \sigma(x) & \leftrightarrow(\tau(x)-\sigma(x)) \geq 0 \leftrightarrow \delta(\tau(x)-\sigma(x))=1 \leftrightarrow \\
& \leftrightarrow 1-\frac{1}{2}(1+\delta(\tau(x)-\sigma(x)))=0 .
\end{aligned}
$$

Hence, we can set

$$
\chi_{[\tau(\vec{x})<\sigma(\vec{x})]}(\vec{x}) \stackrel{\text { def }}{=} \frac{1}{2}(1-\delta(\tau(\vec{x})-\sigma(\vec{x}))) .
$$

We define the bounded minimum operator with the assistance of a $\max _{\tau}$-term in the language of NQA ${ }^{\varnothing}$ as follows:

$$
\operatorname{bmin}_{[\tau(x, \vec{y})=1]}(z, \vec{y}) \stackrel{\text { def }}{=} \max _{[\theta(\tau(x, \vec{y})-1)+\theta(x-(z+1))]}(z, \vec{y}) .
$$

We will also use a common notation $\mu x \leq z[\tau(x, \vec{y})=1](z, \vec{y})$ for above function.

One can show that the above operation has the properties of bounded minimization. Moreover, this bounded minimization operator returns its upper bound plus one, if no suitable $x$ exists. We will use this property later.

Hence, we can introduce the ceiling function as

$$
\lceil y\rceil \stackrel{\text { def }}{=} \mu x \leq \operatorname{li}(y)\left[\chi_{[x \geq y]}(x, y)=1\right](y)
$$

and it is straightforward that the predicate $\mathbb{N}(y)$ is equivalent to $y=\lceil y\rceil$. Using (1) we obtain the characteristic function of predicate $\mathbb{N}(y)$.

For propositional connectives we have

$$
\chi_{[\neg \varphi(\vec{x})]}(\vec{x})=1-\chi_{[\varphi(\vec{x})]}(\vec{x}),
$$

and

$$
\chi_{[\varphi(\vec{x}) \& \psi(\vec{x})]}(\vec{x})=\chi_{[\varphi(\vec{x})]}(\vec{x}) \cdot \chi_{[\psi(\vec{x})]}(\vec{x}) .
$$

We have defined the characteristic function for open formulae. For simplification we denote by $\operatorname{bmin}_{\varphi}(z, \vec{y})$ the expression

$$
\mu x \leq z\left[\chi_{\varphi}(x, \vec{y})=1\right](z, \vec{y}) .
$$

The bounded existential quantifier can be simulated by bounded minimum operator as

$$
(\exists x \leq \sigma(\vec{y})) \varphi(x, \vec{y}) \leftrightarrow\left(\varphi\left(\operatorname{bmin}_{\varphi}(\sigma(\vec{y}), \vec{y}), \vec{y}\right) \& \operatorname{bmin}_{\varphi}(\sigma(\vec{y}), \vec{y}) \leq \sigma(\vec{y})\right) .
$$

We thus put

$$
\chi_{[(\exists x \leq \sigma(\vec{y})) \varphi(x, \vec{y})]}(\vec{y})=\chi_{[\varphi(x, \vec{y}) \& x \leq \sigma(\vec{y}))]}\left(\operatorname{bmin}_{\varphi}(\sigma(\vec{y}), \vec{y}), \vec{y}\right) .
$$

For further work we introduce schemes equivalent to the open minimum scheme:

1. The internal $\Delta_{0}$-induction scheme $\Delta_{0}$-IND 
Axiom $15\left(\Delta_{0}\right.$-IND). Let $\varphi(x, \vec{p})$ be an internal $\Delta_{0}$-formula. Then we have

$$
\varphi(0, \vec{p}) \&(\forall x)(\mathbb{N}(x) \& \varphi(x, \vec{p}) \rightarrow \varphi(x+1, \vec{p})) \rightarrow(\mathbb{N}(y) \rightarrow \varphi(y, \vec{p})) .
$$

2. The open internal bounded minimum scheme O-BMIN for operator* $\operatorname{bmin}_{\varphi}$.

Axiom 16 (O-BMIN). Let $\varphi(x, \vec{p})$ be an open internal formula. Then the term $\operatorname{bmin}_{\varphi}(z, \vec{p})$ satisfies

$$
\begin{aligned}
(\mathbb{N}(y) \& \mathbb{N}(z) & \& y \leq z \& \varphi(y, \vec{p})) \rightarrow \\
& \rightarrow\left(\mathbb{N}\left(\operatorname{bmin}_{\varphi}(z, \vec{p})\right) \& \varphi\left(\operatorname{bmin}_{\varphi}(z, \vec{p}), \vec{p}\right) \& \operatorname{bmin}_{\varphi}(z, \vec{p}) \leq y\right) .
\end{aligned}
$$

LEMMA 2.6. $\mathrm{NQA}^{\varnothing}+\mathrm{O}-\mathrm{MIN}$ is conservative over $\mathrm{NQA}^{\varnothing}+\Delta_{0}-\mathrm{IND}$.

Proof. One can show that the induction axiom for $(\forall u \leq x) \neg \varphi(u)$, where $\varphi$ is an open internal formula,

$$
\begin{array}{r}
(\forall u \leq 0) \neg \varphi(u, \vec{p}) \&(\forall x)(\mathbb{N}(x) \&(\forall u \leq x) \neg \varphi(u, \vec{p}) \rightarrow(\forall v \leq x+1) \neg \varphi(v, \vec{p})) \rightarrow \\
\rightarrow(\forall y)(\mathbb{N}(y) \rightarrow(\forall w \leq y) \neg \varphi(w, \vec{p}))
\end{array}
$$

can be equivalently rewritten as

$$
\begin{aligned}
\neg \varphi(0, \vec{p}) \&(\forall x)(\exists u \leq x)(\forall v \leq x+1)(\mathbb{N}(x) \& \neg \varphi(u, \vec{p}) & \rightarrow \neg \varphi(v, \vec{p})) \rightarrow \\
& \rightarrow(\forall y)(\mathbb{N}(y) \rightarrow \neg \varphi(y, \vec{p})) .
\end{aligned}
$$

Hence, it is equivalent to

$$
\begin{aligned}
(\exists y)(\mathbb{N}(y) \& \varphi(y, \vec{p})) & \rightarrow \varphi(0, \vec{p}) \vee \\
& \vee(\exists x)(\forall u \leq x)(\exists v \leq x+1)(\mathbb{N}(x) \& \neg \varphi(u, \vec{p}) \& \varphi(v, \vec{p})) .
\end{aligned}
$$

By skolemization of the formula

$$
(\forall \vec{p})(\exists x)(\forall u \leq x)(\exists v \leq x+1)(\mathbb{N}(x) \& \neg \varphi(u, \vec{p}) \& \varphi(v, \vec{p})),
$$

we obtain a new function symbols $\min _{\varphi}^{*}(\vec{p})$ and the statements

$$
\begin{aligned}
& (\exists y)(\mathbb{N}(y) \& \varphi(y, \vec{p})) \rightarrow \\
& \quad \rightarrow \varphi(0, \vec{p}) \vee\left(\mathbb{N}\left(\min _{\varphi}^{*}(\vec{p})\right) \&\left(\forall u \leq \min _{\varphi}^{*}\right) \neg \varphi(u, \vec{p}) \& \varphi\left(\min _{\varphi}^{*}+1, \vec{p}\right)\right) .
\end{aligned}
$$

Such a theory is conservative over $\mathrm{NQA}^{\varnothing}+\Delta_{0}$-IND.

It is not too hard to check that by choosing $\min _{\varphi} \stackrel{\text { def }}{=} \min _{\varphi}^{*}-1$ we can prove the O-MIN axiom for any open formula without any min-term occurring recursively.

$$
\text { LEMMA 2.7. NQA } \mathrm{A}^{\varnothing} \text { O-BMIN proves } \Delta_{0} \text {-IND. }
$$

\footnotetext{
*Analogously to the simulation of an existential quantifier by the $\min _{\varphi}$ operator, $\operatorname{bmin}_{\varphi}$ can simulate the bounded existential quantifier but by Lemma 2.5 any internal $\Delta_{0}$-formula is equivalent to an internal open formula. Hence, we don't require an additional condition such as no recursively occurrence of min-term in $\min _{\varphi}$.
} 
Proof. Internal $\Delta_{0}$-formulae are equivalent to open formulae by Lemma 2.5. We prove the internal induction scheme only for open formulae.

Given an open formula $\varphi(x, \vec{p})$ we suppose that there exists $y, \mathbb{N}(y)$, such that $\neg \varphi(y, \vec{p}), \varphi(0, \vec{p})$, and $(\forall x)(\mathbb{N}(x) \& \varphi(x, \vec{p}) \rightarrow \varphi(x+1, \vec{p}))$. Using bounded minimum, we obtain

$$
y_{0}=\operatorname{bmin}_{\neg \varphi(x, \vec{p})}(y, \vec{p}) \leq y .
$$

By O-BMIN axiom we have $\mathbb{N}\left(y_{0}\right)$ and $\neg \varphi\left(y_{0}, \vec{p}\right)$. Obviously $y_{0} \neq 0$ because $\varphi(0, \vec{p})$ and therefore $\mathbb{N}\left(y_{0}-1\right)$. Since $\neg \varphi\left(y_{0}-1, \vec{p}\right)$ implies $y_{0} \leq y_{0}-1$, we have $\varphi\left(y_{0}-1, \vec{p}\right)$. By assumption $(\forall x)(\mathbb{N}(x) \& \varphi(x, \vec{p}) \rightarrow \varphi(x+1, \vec{p}))$ we obtain $\varphi\left(y_{0}, \vec{p}\right)$ which is a contradiction.

LEMMA 2.8. $\mathrm{NQA}^{-}$is a conservative extension of $\mathrm{NQA}^{\varnothing}+\mathrm{O}-\mathrm{BMIN}$.

Proof. By Lemma 2.6 and 2.7 we know that the theory NQA $A^{\varnothing}+$ O-MIN is conservative over NQA ${ }^{\varnothing}+\mathrm{O}-\mathrm{BMIN}$. Note that $\mathrm{NQA}^{\varnothing}+\mathrm{O}-\mathrm{BMIN}$ and NQA ${ }^{\varnothing}+\Delta_{0^{-}}$ IND have the same language.

One can show that $\mathrm{NQA}^{\varnothing}$ also proves the induction for any external formula $\varphi$ in the form

$$
\varphi(0, \vec{p}) \&(\forall x)(\mathbb{N}(x) \& \varphi(x, \vec{p}) \rightarrow \varphi(x+1, \vec{p})) \rightarrow \varphi(\bar{n}, \vec{p}),
$$

where $\bar{n}$ is an arbitrary numeral.

We prove it by induction (in metamathematics) on numeral $n$.

Obviously $\varphi(0, \vec{p}) \rightarrow \varphi(0, \vec{p})$. Let $\bar{n}$ be a numeral such that

$$
\varphi(0, \vec{p}) \&(\forall x)(\mathbb{N}(x) \& \varphi(x, \vec{p}) \rightarrow \varphi(x+1, \vec{p})) \rightarrow \varphi(\bar{n}, \vec{p}) .
$$

Then by $\mathbb{N}(\bar{n})$ and an instance $\varphi(\bar{n}, \vec{p}) \rightarrow \varphi(\bar{n}+1, \vec{p})$, we get $\varphi(\bar{n}+1, \vec{p})$.

Analogously to Lemma 2.6, the external open minimum axiom O-MIN ${ }^{\mathrm{n}}$ is conservative over external $\Delta_{0}$-induction, which is provable in $\mathrm{NQA}^{\varnothing}$.

\section{§3. Consistency of the $\mathrm{NQA}^{-}$theory.}

3.1. An application of the Hilbert-Ackermann Theorem. Recall that the Hilbert-Ackermann Theorem states that the theory is inconsistent if and only if there is a finite set of instances of the axioms that is inconsistent in propositional calculus.

Our aim is to prove the consistency of $\mathrm{NQA}^{-}$formally in PRA. According to Lemma 2.7 it is sufficient to prove

$$
\mathrm{PRA} \vdash \operatorname{Con}\left(\mathrm{NQA}^{\varnothing}+\mathrm{O}-\mathrm{BMIN}\right) .
$$

Recall that the primitive recursive arithmetic (PRA) is a first-order theory with equality whose language contains symbols for all primitive recursive functions. Its axioms contain the recursive definitions of all function symbols, and it has one more inference rule - the open induction rule $(\mathrm{O}-\mathrm{IR})$

$$
\frac{\varphi(0), \varphi(x) \rightarrow \varphi(x+1)}{\varphi(y)}
$$

For consistency proof we will use the Hilbert-Ackermann Theorem whose formalization and proof is possible in primitive recursive arithmetic (see [5]). 
Suppose that NQA $A^{\varnothing}+$ O-BMIN is inconsistent. Using the Hilbert-Ackermann theorem, we get a finite propositionally inconsistent theory $S$. Having substituted any constant of language $N^{\circ} A^{\varnothing}$ (e.g. 1) for free variables in instances of the theory $S$, we can suppose that all axioms of $S$ are closed.

3.2. A finite model $\mathbb{M}(N, \varepsilon)$ with parameters $\mathbf{N}$ and $\varepsilon$. It is straightforward to check that there is an interpretation of the theory of ordered fields OF in PRA (one can use triples $\langle 0 / 1, p, q\rangle$ to encode rational numbers $\pm \frac{p}{q}$ ). The theory of ordered fields is interpretable even in Robinson arithmetic $\mathbb{Q}$ (see [4] for this result).

In order to simplify the notation, let us suppose that we have fixed such an interpretation of OF in PRA, and identify the fractions $+\frac{n}{1}$ with the natural numbers $n$. Then we can consider the field operations to be extensions of arithmetical operations.

Working inside PRA, we will build (i.e., encode in PRA) a finite model $\mathbb{M}(\mathrm{N}, \varepsilon)$ of the language of $S$ dependent on parameters $\mathrm{N}$ and $\varepsilon$. First of all, we construct an assignment ${ }^{*}$ of a function $\tau^{*}$ to every term $\tau$ contained in $S$, and a predicate $\varphi^{*}$ to every open formula $\varphi$ from $S$, by simultaneous recursion on their complexity. The basic field operations and predicates will be interpreted naturally. Note that $\operatorname{bmin}_{\varphi}$ is definable with an assistance of max-term. Then the non-field function symbols are handled as follows:

$$
\begin{aligned}
& \nu_{0}^{*} \stackrel{\text { def }}{=} \mathrm{N}, \\
& (\delta(x)) \stackrel{*}{\text { def }}= \begin{cases}1 & \text { if } x \geq 0 \\
-1 & \text { otherwise }\end{cases} \\
& (n !) \stackrel{\text { def }}{=} \prod_{i=1}^{n-1}(i+1) \\
& \left(x^{n}\right) \stackrel{\text { def }}{=} \prod_{i=0}^{n-1} x \\
& (\operatorname{li}(x)) \stackrel{\text { def }}{=} \begin{cases}\mu y \leq p[y \cdot q \geq p](p, q) & \text { if } x=+\frac{p}{q}, \\
0 & \text { otherwise }\end{cases} \\
& \left(\max _{\tau}(n, \vec{y})\right)^{*} \stackrel{\text { def }}{=} \mu x \leq n\left[(\forall z \leq n)\left(\tau^{*}(z, \vec{y}) \leq \tau^{*}(x, \vec{y})\right](n, \vec{y}),\right. \\
& \left(\sum_{i=0}^{n} \tau(i, \vec{y})\right)^{*} \stackrel{\text { def }}{=} \sum_{i=0}^{n} \tau^{*}(i, \vec{y}) \text {. }
\end{aligned}
$$

Finally, the predicates are interpreted as

$$
\begin{gathered}
(\mathbb{N}(x))^{*} \stackrel{\text { def }}{\equiv} \exists n\left(x=+\frac{n}{1}\right), \\
(\operatorname{Inf}(x))^{*} \stackrel{\text { def }}{=}|x|<\varepsilon .
\end{gathered}
$$

Then we define the universe of our model as

$$
M(\mathrm{~N}, \varepsilon) \stackrel{\text { def }}{=}\{0,1, \mathrm{~N}\} \cup\left\{\tau^{*} ; \tau \in C\right\} .
$$


Predicates from $S$ will be realized in $\mathbb{M}(\mathrm{N}, \varepsilon)$ by the restriction of $(\cdots)^{*}$ to $M(\mathrm{~N}, \varepsilon)$. Similarly, a function symbol $f$ will be realized by the function

$$
f^{\mathbb{M}(\mathrm{N}, \varepsilon)}(\vec{x}) \stackrel{\text { def }}{=} \begin{cases}f^{*}(\vec{x}) & \text { if } f^{*}(\vec{x}) \in M(\mathrm{~N}, \varepsilon), \\ 0 & \text { otherwise. }\end{cases}
$$

Note that $\psi^{*}$ iff $\mathbb{M}(\mathrm{N}, \varepsilon) \vDash \psi$, for any open sentence $\psi$ contained in $S$.

TheOREM 3.1 (PRA). Suppose that $1>\varepsilon>0$ and $1<\mathrm{N}<1 / \varepsilon$, where $\mathrm{N}$ is a natural number (it means it is in form $+\frac{n}{1}$ ). Let $\psi$ be any instance of the axiom of $S$ except for the infinitesimal axioms IF3 and IF5. Then $\mathbb{M}(\mathrm{N}, \varepsilon) \vDash \psi$.

PROOF. It is clear from the definition of $\psi^{*}$ that the instances of the axioms of equality, axioms of ordered fields OF, axioms of Robinson's arithmetic $\mathrm{Q}$, and axiom of sign, power, factorial and li are satisfied in $\mathbb{M}(\mathrm{N}, \varepsilon)$.

Let $\psi$ be an instance of the maximum axiom corresponding to the function symbol $\max _{\tau}(y)$, where $\tau(x)$ is a unary term.* By definition, we have

$$
\max _{\tau}^{*}(0)=\mu x \leq 0\left[(\forall y \leq 0)\left(\tau^{*}(y) \leq \tau^{*}(x)\right)\right]=0,
$$

and

$$
\max _{\tau}^{*}(n+1)= \begin{cases}n+1 & \text { if } \tau^{*}\left(\max _{\tau}^{*}(n)\right)<\tau^{*}(n+1) \\ \max _{\tau}^{*}(n) & \text { if } \tau^{*}\left(\max _{\tau}^{*}(n)\right) \geq \tau^{*}(n+1) .\end{cases}
$$

Similarly, let $\psi$ be an instance of the sum axiom occurring in the theory $S$ and let $\tau(x)$ be the term of the corresponding function symbol $\sum_{i=0}^{n} \tau(i)$. By the definition of $\left(\sum \tau\right)^{*}$ we obtain

$$
\left(\sum_{i=0}^{0} \tau(i)\right)^{*}=\sum_{i=0}^{0} \tau^{*}(i)=\tau^{*}(0)
$$

and also

$$
\left(\sum_{i=0}^{n+1} \tau(i)\right)^{*}=\sum_{i=0}^{n+1} \tau^{*}(i)=\sum_{i=0}^{n} \tau^{*}(i)+\tau^{*}(n+1) .
$$

Let $\psi$ be an instance of O-BMIN for an open internal formula $\varphi(x)$. By definition

$$
\operatorname{bmin}_{\varphi}^{*}(z)=\left(\max _{\theta\left(\chi_{\varphi}(x)-1\right)+\theta(x-(z+1))}(z)\right)^{*}=\mu x \leq z[\phi(x, y, z)](z)
$$

where $\phi(z, y, z)$ is formula $(\forall y \leq z)\left(\theta^{*}\left(\chi_{\varphi}(y)-1\right)+\theta^{*}(y-(z+1)) \leq \theta^{*}\left(\chi_{\varphi}(x)-\right.\right.$ $\left.1)+\theta^{*}(x-(z+1))\right)$ and it is equivalent to $\varphi^{*}(x) \vee(\forall y \leq z) \neg \varphi^{*}(y)$.

Suppose that the assumption $\mathbb{N}^{*}\left(\sigma^{*}\right) \& \mathbb{N}^{*}\left(\tau^{*}\right) \& \sigma^{*} \leq \tau^{*} \& \varphi^{*}\left(\sigma^{*}\right)$ holds. Then clearly

$$
\mathbb{N}^{*}\left(\operatorname{bmin}_{\varphi}^{*}\left(\tau^{*}\right)\right) \& \varphi^{*}\left(\operatorname{bmin}_{\varphi}^{*}\left(\tau^{*}\right)\right) \& \operatorname{bmin}_{\varphi}^{*}\left(\tau^{*}\right) \leq \sigma^{*} .
$$

We have to show that the instance of the infinitesimal axioms except IF3 and IF5 holds in the model $\mathbb{M}(\mathrm{N}, \varepsilon)$ independently on values $\varepsilon$ and $\mathrm{N}$.

According to the assumption of this theorem we have $\mathrm{N}>1$ and thus $\nu_{0}^{*}=$ $\mathrm{N} \neq 0$. From $\mathrm{N}<1 / \varepsilon$ we clearly obtain $\left(\operatorname{Inf}\left(1 / \nu_{0}\right)\right)^{*}$. Furthermore, we suppose that $\mathrm{N}$ is a natural number. It gives us the axiom IF1.

${ }^{*}$ We do not have to consider anything else other than unary terms because all axioms are closed formulae. 
Using the basic properties of the relation $\leq$ we show that the instances of axioms IF2 and IF4 hold independently on the choice of $\varepsilon$ and $\mathrm{N}$.

Suppose $\left|\tau_{1}^{*}\right|<\varepsilon$ and $\left|\tau_{2}^{*}\right| \leq\left|\tau_{1}^{*}\right|$, then $\left|\tau_{2}^{*}\right|<\varepsilon$. Therefore the instances of the axiom IF2 hold.

Let $\left|\tau^{*}\right|<\varepsilon$ then we obtain $\left|\left(1 / \tau^{*}\right)\right|>(1 / \varepsilon)>1>\varepsilon$ and it proves the instances of the axiom IF4.

3.3. The parameters $\mathbf{N}$ and $\varepsilon$. In this section we show how to choose the parameters $\mathrm{N}$ and $\varepsilon$ to satisfy the axioms IF3 and IF5 in model $\mathbb{M}(\mathrm{N}, \varepsilon)$.

LEMMA 3.2. There are natural numbers $0<\mathrm{F}<\mathrm{I}<\mathrm{N}$ such that

1. for any constant term $\tau$ in $S$ we have $\left|1 / \tau^{*}\right|,\left|\tau^{*}\right| \notin[\mathrm{F}, \mathrm{I}]$,

2. $\mathrm{F}^{2}<\mathrm{I}$.

Proof. We define the primitive recursive function

$$
\begin{gathered}
h(0)=0, \\
h(i+1)=h(i)^{2}+1 .
\end{gathered}
$$

Let us denote by $K$ the number of all constant terms in $S$. Choose $\mathrm{N}=$ $h(2 K+1)$. Then the interval $(0, \mathrm{~N}]$ can be divided into $2 K+1$ subintervals as follows

$$
(0, N]=\bigcup_{n=0}^{2 K}(h(n), h(n+1)] .
$$

Then there is $0<j<2 K$ such that $(h(j), h(j+1)] \cap S=\emptyset$. Finally we choose $\mathrm{F}=h(j)$ and $\mathrm{I}=h(j+1)$.

THEOREM 3.3. PRA proves that $\mathrm{NQA}^{-}$is consistent.

Proof. We will work in PRA, and assume that $\mathrm{NQA}^{-}$is inconsistent. Then there is a propositionally inconsistent theory $S$ as described in section 3.1. Define $\mathrm{N}$ as in Lemma 3.2 and $\varepsilon=1 /$ I. If we show that $\mathbb{M}(\mathrm{N}, \varepsilon) \vDash S$, we will obtain a contradiction, because $\mathbb{M}(\mathrm{N}, \varepsilon)$ generates a propositional valuation which satisfies all formulae from $S$.

In view of the Theorem 3.1 it is enough to prove that instances of the axioms IF3 and IF5 from $S$ are satisfied in the model $\mathbb{M}(\mathrm{N}, \varepsilon)$.

Axiom IF3: Let $\tau_{1}, \tau_{2}, \tau_{1} \cdot \tau_{2}$ be constant terms in $S$. Assume $\left|\tau_{1}^{*}\right|<1 / \mathrm{I}$ and $\left|\tau_{2}^{*}\right| \leq \mathrm{F}$, then we obtain

$$
\left|\tau_{1}^{*} \cdot \tau_{2}^{*}\right|<\mathrm{F} / \mathrm{I} \leq 1 / \mathrm{F} .
$$

Hence, we have $1 /\left|\tau_{1}^{*} \cdot \tau_{2}^{*}\right|>\mathrm{F}$ and since $1 /\left|\tau_{1}^{*} \cdot \tau_{2}^{*}\right|$ is not in interval $[\mathrm{F}, \mathrm{I}]$ we get $1 /\left|\tau_{1}^{*} \cdot \tau_{2}^{*}\right|>$ I. Finally it implies $\left|\tau_{1}^{*} \cdot \tau_{2}^{*}\right|<1 /$ I.

Axiom IF5: Let $\tau_{1}, \tau_{2}, \tau_{1}+\tau_{2}$ be constant terms in $S$. Since $\left|\tau_{1}^{*}\right|,\left|\tau_{2}^{*}\right|<\mathrm{F}$ we get

$$
\left|\tau_{1}^{*}+\tau_{2}^{*}\right|<2 \mathrm{~F}<\mathrm{I}
$$

But $\left|\tau_{1}^{*}+\tau_{2}^{*}\right|$ is not in interval $[\mathrm{F}, \mathrm{I}]$, and thus $\left|\tau_{1}^{*}+\tau_{2}^{*}\right|<\mathrm{F}$.

\section{$\S 4$. Interpretations.}


4.1. An interpretation of $\mathrm{I}_{0}+\mathrm{EXP}$ in $\mathrm{NQA}^{-}$. For simplicity we assume that $\mathrm{I} \Delta_{0}+$ EXP is formulated in the language $\left\langle 0,1,+, \cdot, 2^{x},=,<\right\rangle$; its axioms consist of defining equations for these symbols, and the induction schema for all $\Delta_{0}$-formulae in its language. (This is a definable (hence conservative) extension of the usual formulation of $\mathrm{I} \Delta_{0}+$ EXP in the basic arithmetical language $\langle 0, S,+, \cdot,=\rangle$.) Note that the language of $\mathrm{I} \Delta_{0}+$ EXP is contained in the language of $\mathrm{NQA}^{-}$, and all the axioms, except for the bounded induction, are also axioms (or easy theorems) of the theory $\mathrm{NQA}^{-}$, when relativized to the predicate $\mathbb{N}(x)$. We thus define the universe of interpretation to be $\mathbb{N}(x)$, and we leave all functions and predicates absolute.

By Lemma 2.7. we are able to prove the induction for bounded internal (and also arithmetical) formulae from O-BMIN, which we have in NQA'.

COROLlary 4.1. The theory NQA ${ }^{-}$interprets $\mathrm{I} \Delta_{0}+\mathrm{EXP}$.

4.2. An interpretation of $I \Sigma_{1}$ in $\mathrm{NQA}^{+}$. Similarly to the previous interpretation, the language of $I \Sigma_{1}$ is also contained in the language of $\mathrm{NQA}^{+}$. However in this case, the interpretation universe will be only $\mathbb{F} \mathbb{N}(x)$. Note that the predicate $\mathbb{F} \mathbb{N}(x)$ is defined as

$$
\mathbb{F} \mathbb{N}(x) \stackrel{\text { def }}{\equiv} \mathbb{N}(x) \& \neg \operatorname{Inf}\left(\frac{1}{x+1}\right),
$$

and $\mathrm{NQA}^{+}$proves that it is a cut closed under addition and multiplication. Hence, all axioms of $I \Sigma_{1}$ relativized to $\mathbb{F N}(x)$, except for $\Sigma_{1}$-induction, are provable in $\mathrm{NQA}^{-}$.

We want to simulate induction (in this case $\Sigma_{1}$-induction) for arithmetical formulae. The key point is that we can replace every arithmetical $\Sigma_{1}$-formula, with quantifications relativized to $\mathbb{F} \mathbb{N}(x)$, by an open formula, by the following lemma.

Lemma 4.2. Let $\varphi(y, \vec{x})$ be a bounded arithmetical formula. Then in $\mathrm{NQA}^{-}$we have

$$
(\exists y)(\mathbb{F} \mathbb{N}(y) \& \varphi(y, \vec{x})) \leftrightarrow \mathbb{F} \mathbb{N}\left(\mu y \leq \nu_{0}\left(t_{\varphi(y, \vec{x})}(y, \vec{x})=1\right)\right) .
$$

Proof. Assume that $\mathbb{F} \mathbb{N}\left(\mu y \leq \nu_{0}\left(t_{\varphi(y, \vec{x})}(y, \vec{x})=1\right)\right)$ holds. Denote by $y_{0}=$ $\mu y \leq \nu_{0}\left(t_{\varphi(y, \vec{x})}(y, \vec{x})=1\right.$ ), then $\mathbb{F} \mathbb{N}\left(y_{0}\right)$ (in particular, $\left.y_{0}<\nu_{0}\right)$, and by definition (5) from the proof of Lemma 2.5 we have $t_{\varphi\left(y_{0}, x\right)}\left(y_{0}, x\right)=1$. Hence, by Lemma 2.5 , we get $\varphi\left(y_{0}, \vec{x}\right)$. Thus, $y_{0}$ is a witness for the existential formula $(\exists y)(\mathbb{F N}(y) \& \varphi(y, \vec{x}))$.

On the other hand, if $(\mathbb{F} \mathbb{N}(y) \& \varphi(y, \vec{x}))$ holds for some $y$, then $t_{\varphi(y, \vec{x})}(y, \vec{x})=1$, and thus $\mu y \leq \nu_{0}\left(t_{\varphi(y, \vec{x})}(y, \vec{x})=1\right)=y_{0} \leq y$, which implies $\mathbb{F} \mathbb{N}\left(y_{0}\right)$.

Proposition 4.3. The theory $\mathrm{I}_{1}$ is interpretable in $\mathrm{NQA}^{+}$.

Proof. By Lemma 4.2, $\Sigma_{1}$-induction translates to an instance of external open induction in the interpretation described above, hence it follows from $\mathrm{O}-\mathrm{MIN}^{\mathrm{st}}$.

4.3. An interpretation of $W K L_{0}$ in $N Q A^{+}$. We extend the interpretation of $I \Sigma_{1}$ in $\mathrm{NQA}^{+}$to an interpretation of $\mathrm{WKL}_{0}$ in $\mathrm{NQA}^{+}$. The interpretation universe 
for the first-order variables is again $\mathbb{F} \mathbb{N}(x)$, and for the second-order variables it is $\mathbb{N}(X)$. We will interpret the predicate $x \in X$ as

$$
(x \in X)^{*} \stackrel{\text { def }}{\equiv} x \text {-bit }(X)=1,
$$

where

$$
\begin{gathered}
x \text {-bit }(X) \stackrel{\text { def }}{=}\left\lfloor\frac{X}{2^{x}}\right\rfloor-2\left\lfloor\frac{X}{2^{x+1}}\right\rfloor= \begin{cases}1 & \text { if }\left\lfloor\frac{X}{2^{x}}\right\rfloor \text { is odd } \\
0 & \text { if }\left\lfloor\frac{X}{2^{x}}\right\rfloor \text { is even, }\end{cases} \\
\text { and }\left\lfloor\frac{X}{2^{x}}\right\rfloor \stackrel{\text { def }}{=} \mu y \leq \operatorname{li}\left(\frac{X}{2^{x}}\right)\left(\frac{X}{2^{x}}<y+1\right) .
\end{gathered}
$$

We have to verify the interpretation of the $\Sigma_{1}^{0}$-separation schema

$$
(\forall x)(\varphi(x) \rightarrow \neg \psi(x)) \rightarrow(\exists X)(\forall x)((\varphi(x) \rightarrow x \in X) \&(\psi(x) \rightarrow x \notin X)),
$$

for $\Sigma_{1}^{0}$-formulae $\varphi(x)$ and $\psi(x)$ in the language of $\mathrm{WKL}_{0}$.

Assume that $\varphi(x, \vec{p}, \vec{P})$ and $\psi(x, \vec{p}, \vec{P})$ are $\Sigma_{1}^{0}$-formulae (generally with both first-order and second-order parameters) of the form

$$
\begin{aligned}
& \varphi(x, \vec{p}, \vec{P})=(\exists y) \varphi_{0}(y, x, \vec{p}, \vec{P}), \\
& \psi(x, \vec{p}, \vec{P})=(\exists y) \psi_{0}(y, x, \vec{p}, \vec{P}),
\end{aligned}
$$

where $\varphi_{0}$ and $\psi_{0}$ are $\Delta_{0}^{0}$-formulae. Suppose that $((\forall x)(\varphi(x) \rightarrow \neg \psi(x)))^{*}$. Set

$$
\begin{gathered}
y_{0}(x) \stackrel{\text { def }}{=} \mu y \leq \nu_{0}\left(\varphi_{0}^{*}(y, x) \vee \psi_{0}^{*}(y, x)\right), \\
X \stackrel{\text { def }}{=} \sum_{x=0}^{\nu_{0}-1} 2^{x} \cdot t_{\varphi_{0}^{*}(y, x)}\left(y_{0}(x), x\right) .
\end{gathered}
$$

Obviously $X<2^{\nu_{0}}$. We will verify that $X$ satisfies the condition of separation.

Suppose $\mathbb{F} \mathbb{N}(x)$ and $\varphi^{*}(x)$, then by Lemma 4.2 we have $\mathbb{F} \mathbb{N}\left(y_{0}(x)\right)$, and by Lemma 2.5 we have $t_{\varphi_{0}^{*}(y, x)}\left(y_{0}(x), x\right)=1$. Thus $(x \in X)^{*}$.

On the other hand, let us assume that $\mathbb{F} \mathbb{N}(x)$ and $\psi^{*}(x)$. Hence $\mathbb{F} \mathbb{N}\left(y_{0}(x)\right)$, and since $\psi^{*}(x)$ implies $\neg \varphi^{*}(x)$ (from assumption $\left.((\forall x)(\varphi(x) \rightarrow \neg \psi(x)))^{*}\right)$, using Lemma 2.5 we obtain $t_{\varphi_{0}^{*}(y, x)}\left(y_{0}(x), x\right)=0$. Therefore $(x \notin X)^{*}$.

4.4. An interpretation of $\mathrm{NQA}^{-}$in $\mathrm{I}_{0}+$ EXP. We start with an interpretation of $\mathrm{NQA}^{-}$in an auxiliary theory $T$. The language of $T$ contains the language of $\mathrm{I} \Delta_{0}+\mathrm{EXP}$, and additionally

1. a unary predicate $\mathbb{F} \mathbb{N}(x)$, and

2. a new constant $\nu_{0}$.

The axioms of the theory consists of

1. the axioms of $\mathrm{I} \Delta_{0}+\mathrm{EXP}$,

2. axioms expressing that $\mathbb{F} \mathbb{N}(x)$ is a proper cut closed under multiplication (i.e., $\mathbb{F} \mathbb{N}(0), \mathbb{F} \mathbb{N}(x) \rightarrow \mathbb{F} \mathbb{N}(x+1), \mathbb{F} \mathbb{N}(x) \& \mathbb{F} \mathbb{N}(y) \rightarrow \mathbb{F} \mathbb{N}(x+y) \& \mathbb{F} \mathbb{N}(x \cdot y)$, $x \leq y \& \mathbb{F} \mathbb{N}(y) \rightarrow \mathbb{F} \mathbb{N}(x)$, and $\left.\neg \mathbb{F} \mathbb{N}\left(\nu_{0}\right)\right)$.

LEMMA 4.4. $\mathrm{NQA}^{-}$is interpretable in $T$. 
Proof. As in the Section 3.2, we extend the theory $T$ by rational numbers. We denote them as usual by $\pm \frac{p}{q}$. In such an extended theory we can interpret all functions and predicates of $\mathrm{NQA}^{-}$. Recall that $T$ extends $\mathrm{I} \Delta_{0}+\mathrm{EXP}$, and that all Kalmár elementary recursive functions have well-behaved bounded definitions in the latter theory, hence we can use them freely.

The universe of our interpretation * will be the universe of rational numbers $\mathbb{Q}(x)$. The field operations and predicates will be the same as in the case of rationals. We have to show how to interpret non-field functions, and predicates.

$$
\begin{gathered}
\left(x^{n}\right)^{*}=\prod_{i=0}^{n-1} x^{*}, \\
(n !)^{*}=\prod_{i=0}^{n-1}(i+1), \\
\left(\delta\left( \pm \frac{p}{q}\right)\right)^{*}= \pm 1, \\
\left(\operatorname{li}\left( \pm \frac{p}{q}\right)\right)^{*}=p \\
(\mathbb{N}(x))^{*} \stackrel{\text { def }}{=} \exists n\left(x=+\frac{n}{1}\right), \\
\left(\sum_{i=0}^{n} \tau(i)\right)^{*}=\sum_{i=0}^{n} \tau^{*}(i),\left(\max _{\tau}(n)\right)^{*}=\mu y \leq n\left((\forall x \leq n)\left(\tau^{*}(x) \leq \tau^{*}(y)\right)\right), \\
\left(\operatorname{Inf}\left( \pm \frac{p}{q}\right)\right)^{*} \stackrel{\text { def }}{\equiv}(p \neq 0) \&(\forall x)\left(\mathbb{F} \mathbb{N}(x) \rightarrow x<\left| \pm \frac{q}{p}\right|\right), \\
\left(\min _{\varphi}(\vec{x})=y\right)^{*} \stackrel{\text { def }}{\equiv}((\mathbb{N}(y) \& \varphi(y, \vec{x}) \&(\forall z<y)(\mathbb{N}(z) \rightarrow \neg \varphi(z, \vec{x}))) \vee \\
\left.\vee\left(y=0 \&\left(\exists y^{\prime}\right)\left(\mathbb{N}\left(y^{\prime}\right) \& \varphi\left(y^{\prime}, \vec{x}\right) \&\left(\forall z<y^{\prime}\right)(\mathbb{N}(z) \rightarrow \neg \varphi(z, \vec{x}))\right)\right)\right)^{*} .
\end{gathered}
$$

It is easy to show that all axioms of $\mathrm{NQA}^{-}$, except for minimization schemata, are provable in $T$. Note that for an internal open formula $\varphi,(\varphi(y, \vec{x}))^{*}$ is equivalent to a bounded formula and therefore internal minimization schema follows from $\Delta_{0}$-induction.

Suppose that $\varphi(y, \vec{x})$ is an external open formula, and $\bar{n}$ is a numeral such that $\varphi^{*}(\bar{n}, \vec{x})$ holds. Then we can search through

$$
\varphi^{*}(\overline{0}, \vec{x}), \varphi^{*}(\overline{1}, \vec{x}), \ldots, \varphi^{*}(\bar{n}, \vec{x})
$$

to find the least $i \leq n$ satisfying $\varphi^{*}(\bar{i}, \vec{x})$.

We shall make use of the following well-known (but not well-documented) result. We advise the reader to consult [14] for background on general properties of interpretations.

LEMMA 4.5 (folklore). Let $S$ be a recursively axiomatized extension of $\mathrm{I} \Delta_{0}+$ EXP. Then $S$ interprets $S+\neg \operatorname{Con}^{C F}(S)$, where $C o n^{C F}(S)$ denotes the formalized cut-free consistency of $S$.

Proof (Sketch). Put $U=S+\neg \operatorname{Con}^{\mathrm{CF}}(S)$. By formalized Gödel's Second Incompleteness Theorem (which holds for cut-free provability, using the fact that $\mathrm{I} \Delta_{0}+$ EXP proves elimination of cuts with standard cut-rank), we have

$$
S+\mathrm{Con}^{\mathrm{CF}}(S) \vdash \mathrm{Con}^{\mathrm{CF}}(U) .
$$


Since

$$
\mathrm{Q}+\operatorname{Con}^{\mathrm{CF}}(U) \text { interprets } U
$$

by the Interpretation Existence Lemma (proved for PA by Feferman [3]; the strengthening to $Q$ is, again, folklore, using Solovay's method of shortening of definable cuts), we get

$$
S+\operatorname{Con}^{\mathrm{CF}}(S) \text { interprets } U \text {. }
$$

Trivially

$$
S+\neg \mathrm{Con}^{\mathrm{CF}}(S) \text { interprets } U,
$$

and combining these two interpretations yields the result.

LEMma 4.6. The theory $T$ is interpretable in $\mathrm{I} \Delta_{0}+\mathrm{EXP}$.

Proof. The theory $\mathrm{I} \Delta_{0}+$ EXP is well-known to be finitely axiomatizable and sequential (see e.g., [5]), hence by a result of Pudlák [8], there exists an $\mathrm{I} \Delta_{0}+$ EXP-definable cut $J(x)$, provably closed under multiplication, such that

$$
\mathrm{I} \Delta_{0}+\mathrm{EXP} \vdash\left(\mathrm{Con}^{\mathrm{CF}}\left(\mathrm{I} \Delta_{0}+\mathrm{EXP}\right)\right)^{J} .
$$

Let us define

$$
\begin{aligned}
\mathbb{F} \mathbb{N}(x) & \stackrel{\text { def }}{=} J(x), \\
\nu_{0} & \stackrel{\text { def }}{=} \text { the least code of a cut-free proof of inconsistency in } \mathrm{I} \Delta_{0}+\text { EXP. }
\end{aligned}
$$

Clearly, this is an interpretation of $T$ in $\mathrm{I} \Delta_{0}+\mathrm{EXP}+\neg \mathrm{Con}^{\mathrm{CF}}\left(\mathrm{I} \Delta_{0}+\mathrm{EXP}\right)$, which is interpretable in $\mathrm{I} \Delta_{0}+$ EXP by Lemma 4.5 .

Corollary 4.7. There is an interpretation of $\mathrm{NQA}^{-}$in $\mathrm{I} \Delta_{0}+\mathrm{EXP}$.

\section{REFERENCES}

[1] David Ballard and Karel HrbáČEK, Standard foundations for nonstandard analysis, The Journal of Symbolic Logic, vol. 57 (1992), no. 2, pp. 741-748.

[2] Rolando Chuaqui and Patrick Suppes, Free-variable axiomatic foundations of infinitesimal analysis: A fragment with finitary consistency proof, The Journal of Symbolic Logic, vol. 60 (1995), no. 1, pp. 122-159.

[3] Solomon Feferman, Arithmetization of metamathematics in a general setting, Fundamenta Mathematicae, vol. 49 (1960), pp. 35-92.

[4] António M. Fernandes and Fernando Ferreira, Groundwork for weak analysis, The Journal of Symbolic Logic, vol. 67 (2002), no. 2, pp. 557-578.

[5] Petr Hájek and Pavel Pudlák, Metamathematics of first-order arithmetic, Springer-Verlag, 1993.

[6] JAN Mycielski, Locally finite theories, The Journal of Symbolic Logic, vol. 51 (1986), pp. 59-62.

[7] Edward Nelson, Internal set theory: A new approach to nonstandard analysis, Bulletin of the American Mathematical Society, vol. 83 (1977), pp. 1165-1198.

[8] PAVel PudLÁK, Cuts, consistency statements and interpretability, The Journal of Symbolic Logic, vol. 50 (1985), pp. 423-441.

[9] Abraham Robinson, Non-standard analysis, North-Holland Publishing Company, 1966.

[10] Joseph R. Shoenfield, Mathematical logic, Addison-Wesley Publishing Company, 1967.

[11] Antonín Sochor, Klasická matematická logika, Karolinum, Prague, 2001, in Czech. 
[12] Patrick Suppes and Rolando Chuaqui, A finitarily consistent free-variable positive fragment of infinitesimal analysis, Proceedings of the IX. Latin American Symposium on Mathematical Logic, 1993, pp. 1-59.

[13] William W. Tait, Finitism, Journal of Philosophy, vol. 78 (1981), pp. 524-564.

[14] Albert Visser, An overview of interpretability logic, Advances in modal logic '96 (M. Kracht, M. de Rijke, H. Wansing, and M. Zakharyaschev, editors), CSLI, Stanford, 1998, pp. 307-359.

[15] Petr Vopěnka, Mathematics in the Alternative Set Theory, Teubner, Leipzig, 1979.

[16] RichaRD ZACH, The practice of finitism: Epsilon calculus and consistency proofs in Hilbert's program, Syntese, vol. 137 (2003), pp. 211-259.

MATHEMATICAL INSTITUTE

CZECH ACADEMY OF SCIENCES

PRAGUE, 115 67, CZECH REPUBLIC

E-mail: rossler@math.cas.cz

DEPARTMENT OF COMPUTER SCIENCE9

UNIVERSITY OF TORONTO

TORONTO, ONTARIO M5S 3G4

E-mail: jerabek@math.cas.cz 